\title{
Strange matter and kaon to pion ratio in the SU(3) Polyakov-Nambu-Jona-Lasinio model
}

\author{
A. V. Friesen, Yu. L. Kalinovsky, and V. D. Toneev \\ Bogoliubov Laboratory of Theoretical Physics (BLTP), Joint Institute for Nuclear Research, 141982 Dubna, Russia
}

(Received 6 September 2018; published 2 April 2019)

\begin{abstract}
The behavior of strange matter in the frame of the SU(3) Polyakov-loop extended Nambu-Jona-Lasinio (PNJL) model including the $U_{A}(1)$ anomaly is considered. We discuss the appearance of a peak in the ratio of the number of strange mesons to nonstrange mesons known as the "horn." The PNJL model gives a schematic description of the chiral phase transition and meson properties at finite temperature and density. By using the model, we can show that the splitting of kaon and antikaon masses appears as a result of the introduction of density. This may explain the difference in the $K^{+} / \pi^{+}$ratio and the $K^{-} / \pi^{-}$ratio at low $\sqrt{s_{N N}}$ and their tendency to the same value at high $\sqrt{s_{N N}}$. We also show that the rise in the ratio $K^{+} / \pi^{+}$appears near the critical endpoint when we build the $K^{+} / \pi^{+}$ratio along the phase-transition diagram and it can be considered as a critical region signal.
\end{abstract}

DOI: 10.1103/PhysRevC.99.045201

\section{INTRODUCTION}

The study of matter formed during the collision of heavy ions at high energies is currently of significant interest in highenergy physics. Much interest still focuses on the search of the critical endpoint and phase transition in hot and dense matter. The search for quark-gluon plasma (QGP) where hadrons dissolve into interacting gluons and quarks is difficult due to the short lifetime of the QCD phase. It is needed to find sensible probes for the transition to the QGP phase (i.e., deconfinement transition and the chiral symmetry restoration). One of the suggested signals was the strangeness enhancement which was explained through the interactions between partons in QGP.

Intriguing results were obtained from $\mathrm{PbPb}$ and $\mathrm{AuAu}$ collisions: a structure in the ratio of the positive charged kaon to the positive charged pion, named the "horn" (see Fig. 1), was found. The horn was first described by the NA49 Collaboration [1] and the work aroused significant interest. Investigations at energies from 7 to $200 \mathrm{AGeV}$ were made by the (Relativistic Heavy Ion Collider Beam Energy Scan) [2] and it was shown that the data can be placed on the same curve [1,3-5]. Such enhancement was also observed in the ratio of other positive charged strange particles to the positive charged nonstrange pions. At that time, the horn was not observed in the ratio of negative charged particles $K^{-} / \pi^{-}$[6]. In the $p+p$ collision, the $K^{+} / \pi^{+}$ratio shows smooth behavior $[3,6,7]$.

Published by the American Physical Society under the terms of the Creative Commons Attribution 4.0 International license. Further distribution of this work must maintain attribution to the author(s) and the published article's title, journal citation, and DOI. Funded by $S C O A P^{3}$.
The strangeness suppression in $p p$ collisions can result from insufficient time and space of colliding and the impossibility of reaching the statistical equilibrium of strange flavor with light quarks.

An exact theoretical reproduction of the horn in the $K / \pi$ ratio still does not exist. The microscopic transport model that includes only the hadron phase and does not include the quark-gluon phase cannot reproduce experimental data [8-12] and, as a result, in the works $[3,13]$ the authors suggest that such a peak in the ratio can be explained as the onset of deconfinement.

The statistical model of early stages (SMES) considers a slow increase and the following jump in the ratio of strangeto-nonstrange particle production as a result of the deconfinement transition. According to the SMES, at low collision energies confined matter is produced, and the increase in the ratio is due the low $T$ of the early stage and the large mass of strange particles. When the deconfinement transition occurs the strange quarks mass tends to its current mass $\left(m_{s}<T\right)$ and the strangeness yield becomes independent of energy in the QGP (the "jump" from a high value of the ratio to its constant value) [13].

Success in the description of experimental data was achieved when the partial restoration of chiral symmetry [14] was added in the transport model. In the work the primary interaction was described through the mechanism of excitation and decay of color objects-strings. The function of the string fragmentation includes the dependence on the baryon density, thus modelling the mechanism of the partial chiral symmetry restoration $[14,15]$. The authors showed that partial chiral symmetry restoration is responsible for the quick increase in the $K^{+} / \pi^{+}$ratio at low energies and its decrease with increasing energy (they explained this decrease as a result of chiral condensate destruction).

The qualitative reproduction of the peak in the energy dependence of the kaon to pion ratio was obtained in the statistical model, which includes hadron resonances and the $\sigma$ 


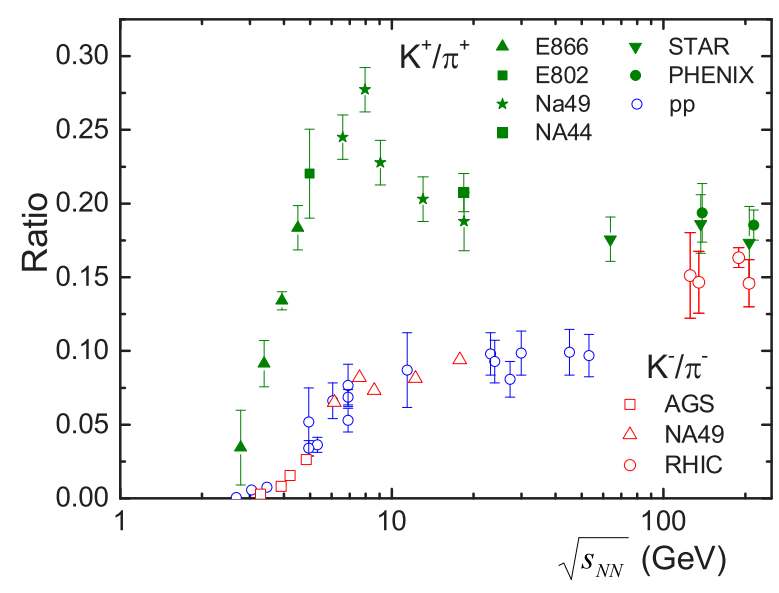

FIG. 1. The $K^{+} / \pi^{+}$and $K^{-} / \pi^{-}$ratio as the $\sqrt{s_{N N}}$ function for $\mathrm{Pb}+\mathrm{Pb}$ and $\mathrm{Au}+\mathrm{Au}$ central collisions [1,3-5]. Blue circles are the $K^{+} / \pi^{+}$ratio for $p p$ collisions.

meson. This type of model implies the existence of the critical temperature for hadrons, which plays the role of the hadron phase transition [16].

The SU(3) Nambu-Jona-Lasinio (NJL) model with the Polyakov loop (PNJL model) seems to be most promising as an instrument for describing the chiral phase transition, the deconfinement properties, and the existence of quarks and hadron states [17-19]. The chiral symmetry breaking in the model is explained through a mechanism of adding the chiral condensate to the current quark. The Polyakov loop extended model in addition to the chiral transition takes into account the deconfinement transition which is described by the Polyakov loop. The phase diagram of the PNJL model corresponds to its modern concept: at low temperature and high chemical potential the system suffers a first-order phase transition. At high temperature and low chemical potential in system the chiral phase transition line is a crossover [17,19]. The disadvantage of the model is that the critical temperature of the crossover transition at low chemical potential in the PNJL model is higher than in lattice QCD, $T_{c}=154$ (9) [20], and the temperature of the critical endpoint (CEP) is lower than in other models [20-22].

We address this paper to the problem of the kaon to pion ratio in the context of the SU(3) PNJL model. In Sec. II the formalism of the PNJL model and the behavior of mesons and quarks at zero chemical potential is discussed. The PNJL model generalized to finite chemical potential is presented in Sec. III. In Sec. IV, the results are discussed and conclusions are given.

\section{MODEL FORMALISM}

We consider the Polyakov-loop extended SU(3) NambuJona-Lasinio model with scalar-pseudoscalar interaction and the t'Hooft interaction, which breaks $\mathrm{U}_{A}(1)$ symmetry $[18,19]$. The global $\mathrm{SU}(3) \otimes \mathrm{SU}(3)$ chiral symmetry of the Lagrangian is obviously broken upon introducing the nonzero current quark masses $\hat{m}=\operatorname{diag}\left(m_{u}, m_{d}, m_{s}\right)$, and the confinement and deconfinement properties $\left(\mathrm{Z}_{3}\right.$ symmetry $)$ are described by the effective potential $\mathcal{U}(\Phi, \bar{\Phi} ; T)$ :

$$
\begin{aligned}
\mathcal{L}= & \bar{q}\left(i \gamma^{\mu} D_{\mu}-\hat{m}-\gamma_{0} \mu\right) q \\
& +\frac{1}{2} g_{S} \sum_{a=0}^{8}\left[\left(\bar{q} \lambda^{a} q\right)^{2}+\left(\bar{q} i \gamma_{5} \lambda^{a} q\right)^{2}\right] \\
& +g_{D}\left\{\operatorname{det}\left[\bar{q}\left(1+\gamma_{5}\right) q\right]+\operatorname{det}\left[\bar{q}\left(1-\gamma_{5}\right) q\right]\right\} \\
& -\mathcal{U}(\Phi, \bar{\Phi} ; T),
\end{aligned}
$$

where $q=(u, d, s)$ is the quark field with three flavors, $N_{f}=$ 3 , and three colors, $N_{c}=3, \lambda^{a}$ are the Gell-Mann matrices, $a=0,1, \ldots, 8, \lambda^{0}=\sqrt{\frac{2}{3}} \mathbf{I}$ and $\mathrm{D}_{\mu}=\partial^{\mu}-i A^{\mu}$, where $A^{\mu}$ is the gauge field with $A^{0}=-i A_{4}$ and $A^{\mu}(x)=g_{S} A_{a}^{\mu} \frac{\lambda_{a}}{2}$ absorbs the strong interaction coupling.

The effective potential $U(\Phi, \bar{\Phi} ; T)$ depends on temperature $T$, the Polyakov loop field $\Phi$ and its complex conjugate $\bar{\Phi}$, which can be obtained through the expectation value of the Polyakov line [23,24]:

$$
\Phi(\vec{x})=\frac{1}{N_{c}} \operatorname{Tr}_{c}\langle\langle L(\vec{x})\rangle\rangle,
$$

where

$$
L(\vec{x})=\mathcal{P} \exp \left[i \int_{0}^{\beta} d \tau A_{4}(\vec{x}, \tau)\right]
$$

The Polyakov loop field is the order parameter for $Z_{3}$ symmetry restoration, which is restored as $\Phi \rightarrow 0$ (confinement) and broken as $\Phi \rightarrow 1$ (deconfinement) [25]. The effective potential $U(\Phi, \bar{\Phi} ; T)$ has to reproduce lattice QCD data in the gauge sector [26] and must satisfy the $Z_{3}$ center symmetry. Based on these suggestions one can choose any form of the potential $[17,24,27]$. In this work the following general polynomial form is used [24]:

$$
\begin{aligned}
\frac{\mathcal{U}(\Phi, \bar{\Phi} ; T)}{T^{4}} & =-\frac{b_{2}(T)}{2} \bar{\Phi} \Phi-\frac{b_{3}}{6}\left(\Phi^{3}+\bar{\Phi}^{3}\right)+\frac{b_{4}}{4}(\bar{\Phi} \Phi)^{2} \\
b_{2}(T) & =a_{0}+a_{1}\left(\frac{T_{0}}{T}\right)+a_{2}\left(\frac{T_{0}}{T}\right)^{2}+a_{3}\left(\frac{T_{0}}{T}\right)^{3}
\end{aligned}
$$

For the effective potential the following parameters were chosen: $T_{0}=0.19 \mathrm{GeV}, a_{0}=6.75, a_{1}=-1.95, a_{2}=2.625$, $a_{3}=-7.44, b_{3}=0.75, b_{4}=7.5$ [24]. The grand potential density for the PNJL model in the mean-field approximation can be obtained from the Lagrangian density(1) [28]:

$$
\begin{aligned}
\Omega= & U(\Phi, \bar{\Phi} ; T)+g_{S} \sum_{i=u, d, s}\left\langle\bar{q}_{i} q_{i}\right\rangle^{2}+4 g_{D}\left\langle\bar{q}_{u} q_{u}\right\rangle \\
& \times\left\langle\bar{q}_{d} q_{d}\right\rangle\left\langle\bar{q}_{s} q_{s}\right\rangle-2 N_{c} \sum_{i=u, d, s} \int \frac{d^{3} p}{(2 \pi)^{3}} E_{i} \\
& -2 T \sum_{i=u, d, s} \int \frac{d^{3} p}{(2 \pi)^{3}}\left[N_{\Phi}^{+}\left(E_{i}\right)+N_{\Phi}^{-}\left(E_{i}\right)\right],
\end{aligned}
$$


with the functions

$$
\begin{aligned}
N_{\Phi}^{+}\left(E_{i}\right) & =\operatorname{Tr}_{\mathrm{c}}\left[\ln \left(1+L^{\dagger} e^{-\beta\left(E_{i}-\mu\right)}\right)\right] \\
& =\left[1+3\left(\Phi+\bar{\Phi} e^{-\beta E_{i}^{+}}\right) e^{-\beta E_{i}^{+}}+e^{-3 \beta E_{i}^{+}}\right], \\
N_{\Phi}^{-}\left(E_{i}\right) & =\operatorname{Tr}_{\mathrm{c}}\left[\ln \left(1+L e^{-\beta\left(E_{p}+\mu\right)}\right)\right] \\
& =\left[1+3\left(\bar{\Phi}+\Phi e^{-\beta E_{p}^{-}}\right) e^{-\beta E_{p}^{-}}+e^{-3 \beta E_{p}^{-}}\right],
\end{aligned}
$$

where $E_{i}^{ \pm}=E_{i} \mp \mu_{i}, \beta=1 / T, E_{\mathrm{i}}=\left(\mathbf{p}_{\mathbf{i}}{ }^{2}+m_{i}^{2}\right)^{1 / 2}$ is the energy of quarks, and $\left\langle\bar{q}_{i} q_{i}\right\rangle$ is the quark condensate.
To obtain the value of the Polyakov loop field $\Phi, \bar{\Phi}$, one needs to minimize the grand potential over its parameters

$$
\frac{\partial \Omega}{\partial \Phi}=0, \quad \frac{\partial \Omega}{\partial \bar{\Phi}}=0 .
$$

The gap equation for quarks depends on the quark condensates:

$$
m_{i}=m_{0 i}-2 g_{S}\left\langle\bar{q}_{i} q_{i}\right\rangle-2 g_{D}\left\langle\bar{q}_{j} q_{j}\right\rangle\left\langle\bar{q}_{k} q_{k}\right\rangle,
$$

where $i, j, k=\mathrm{u}, \mathrm{d}, \mathrm{s}$ are chosen in cyclic order. The quark condensate is $[18,28]$

$$
\left\langle\bar{q}_{i} q_{i}\right\rangle=i \int \frac{d p}{(2 \pi)^{4}} \operatorname{Tr} S\left(p_{i}\right)=-2 N_{c} \int \frac{d^{3} p}{(2 \pi)^{3}} \frac{m_{i}}{E_{i}}\left[1-f_{\Phi}^{+}\left(E_{i}\right)-f_{\Phi}^{-}\left(E_{i}\right)\right],
$$

with the modified Fermi functions:

$$
\begin{aligned}
& f_{\Phi}^{+}\left(E_{\mathrm{p}}-\mu\right)=\frac{\bar{\Phi} e^{-\beta\left(E_{\mathrm{p}}-\mu\right)}+2 \Phi e^{-2 \beta\left(E_{\mathrm{p}}-\mu\right)}+e^{-3 \beta\left(E_{\mathrm{p}}-\mu\right)}}{1+3\left(\bar{\Phi}+\Phi e^{-\beta\left(E_{\mathrm{p}}-\mu\right)}\right) e^{-\beta\left(E_{\mathrm{p}}-\mu\right)}+e^{-3 \beta\left(E_{\mathrm{p}}-\mu\right)}}, \\
& f_{\Phi}^{-}\left(E_{\mathrm{p}}+\mu\right)=\frac{\Phi e^{-\beta\left(E_{\mathrm{p}}+\mu\right)}+2 \bar{\Phi} e^{-2 \beta\left(E_{\mathrm{p}}+\mu\right)}+e^{-3 \beta\left(E_{\mathrm{p}}+\mu\right)}}{1+3\left(\Phi+\bar{\Phi} e^{-\beta\left(E_{\mathrm{p}}+\mu\right)}\right) e^{-\beta\left(E_{\mathrm{p}}+\mu\right)}+e^{-3 \beta\left(E_{\mathrm{p}}+\mu\right)}} .
\end{aligned}
$$

The meson masses in NJL-like models are defined by the Bethe-Salpeter equation at $\mathbf{P}=0$ [29]:

$$
1-P_{i j} \Pi_{i j}^{P}\left(P_{0}=M, \mathbf{P}=\mathbf{0}\right)=0,
$$

where for nondiagonal pseudoscalar mesons $\pi, K$,

$$
\begin{aligned}
& P_{\pi}=g_{S}+g_{D}\left\langle\bar{q}_{s} q_{s}\right\rangle, \\
& P_{K}=g_{S}+g_{D}\left\langle\bar{q}_{u} q_{u}\right\rangle,
\end{aligned}
$$

and the polarization operator has the form

$$
\Pi_{i j}^{P}\left(P_{0}\right)=4\left\{\left(I_{1}^{i}+I_{1}^{j}\right)-\left[P_{0}^{2}-\left(m_{i}-m_{j}\right)^{2}\right] I_{2}^{i j}\left(P_{0}\right)\right\},
$$

where integrals $I_{1}^{i} I_{2}^{i j}\left(P_{0}\right)$ are defined as

$$
\begin{aligned}
I_{1}^{i} & =i N_{c} \int \frac{d^{4} p}{(2 \pi)^{4}} \frac{1}{p^{2}-m_{i}^{2}}, \\
I_{2}^{i j}\left(P_{0}\right) & =i N_{c} \int \frac{d^{4} p}{(2 \pi)^{4}} \frac{1}{\left(p^{2}-m_{i}^{2}\right)\left[\left(p+P_{0}\right)^{2}-m_{j}^{2}\right]},
\end{aligned}
$$

with the quark energy $E_{i, j}=\left(\mathrm{p}^{2}+m_{i, j}^{2}\right)^{1 / 2}$. When the meson mass exceeds the total value of its consistent $P_{0}>m_{i}+m_{j}$, the meson turns into the resonance state. In this case, the complex properties of the integrals have to be taken into account and the solution has to be defined in the form $P_{0}=M_{M}-\frac{1}{2} i \Gamma_{M}$. Each equation (12) splits into two equations from which the meson mass $M_{M}$ and the meson width $\Gamma_{M}$ can be obtained [30].

The mass spectrum for zero chemical potential is shown in Fig. 2. The following set of parameters was chosen for calculations: the current quark masses $m_{0 u}=m_{0 d}=5.5 \mathrm{MeV}$, $m_{0 s}=0.131 \mathrm{GeV}$, the cutoff $\Lambda=0.652 \mathrm{GeV}$, couplings $g_{D}=$ $89.9 \mathrm{GeV}^{-} 2$ and $g_{S}=4.3 \mathrm{GeV}^{-5}$. As can be seen from Fig. 2, at zero chemical potential charged multiplets are degenerate. The $q \bar{q}$ threshold for the mesons is defined as $2 m_{u}$ for pion and $m_{u}+m_{s}$ for kaon. The temperature at which the meson mass becomes equal to the value of the $q \bar{q}$ threshold is the Mott temperature $T_{\text {Mott }}^{\pi}$. After the Mott temperature the meson from the bound state turns into the resonance state and can dissociate into its constituents. As can be seen, the pion and kaon in the PNJL model decay at a near temperature $\left(T_{\text {Mott }}^{\pi}=\right.$ $0.232, T_{\text {Mott }}^{K}=0.23 \mathrm{GeV}$ ).

The normalized quark condensate of light and strange quarks and the Polyakov loop field $\Phi$ are shown in the bottom panel Fig. 2. The chiral condensate is the order parameter of the spontaneous chiral symmetry restoration in the model, and when the temperature exceeds a characteristic transition temperature, the condensate "melts" $(\langle q \bar{q}\rangle \rightarrow 0)$, the mass of quarks tends to their current values and the chiral symmetry is restored. At that time, the Polyakov loop field $\Phi \rightarrow 1$ and it is the signal that $Z_{3}$-symmetry breaking and deconfinement occurs. As can be seen, the strange quark condensate is very high and the chiral symmetry restoration in the strange sector does not occur.

\section{FINITE BARYON DENSITY}

The calculations in SU(3) NJL-like models are complicated by the need to introduce the strange quark chemical potential. As a rule, when only thermodynamics is considered, the chemical potential of the strange quark is supposed equal to zero, $\mu_{s}=0 \mathrm{GeV}$. We consider the following two cases:

Case I: matter with equal chemical potentials $\mu_{u}=\mu_{d}=$ $\mu_{s}$;

Case II: matter with $\mu_{u}=\mu_{d}$ and $\mu_{s}=0.55 \mu_{u}$.

For both cases the phase diagrams are similar and are shown in Fig. 3 (top panel). The phase diagram also has a structure similar to the SU(2) NJL case [21,22]: at high temperature and low density (chemical potential) the phase 

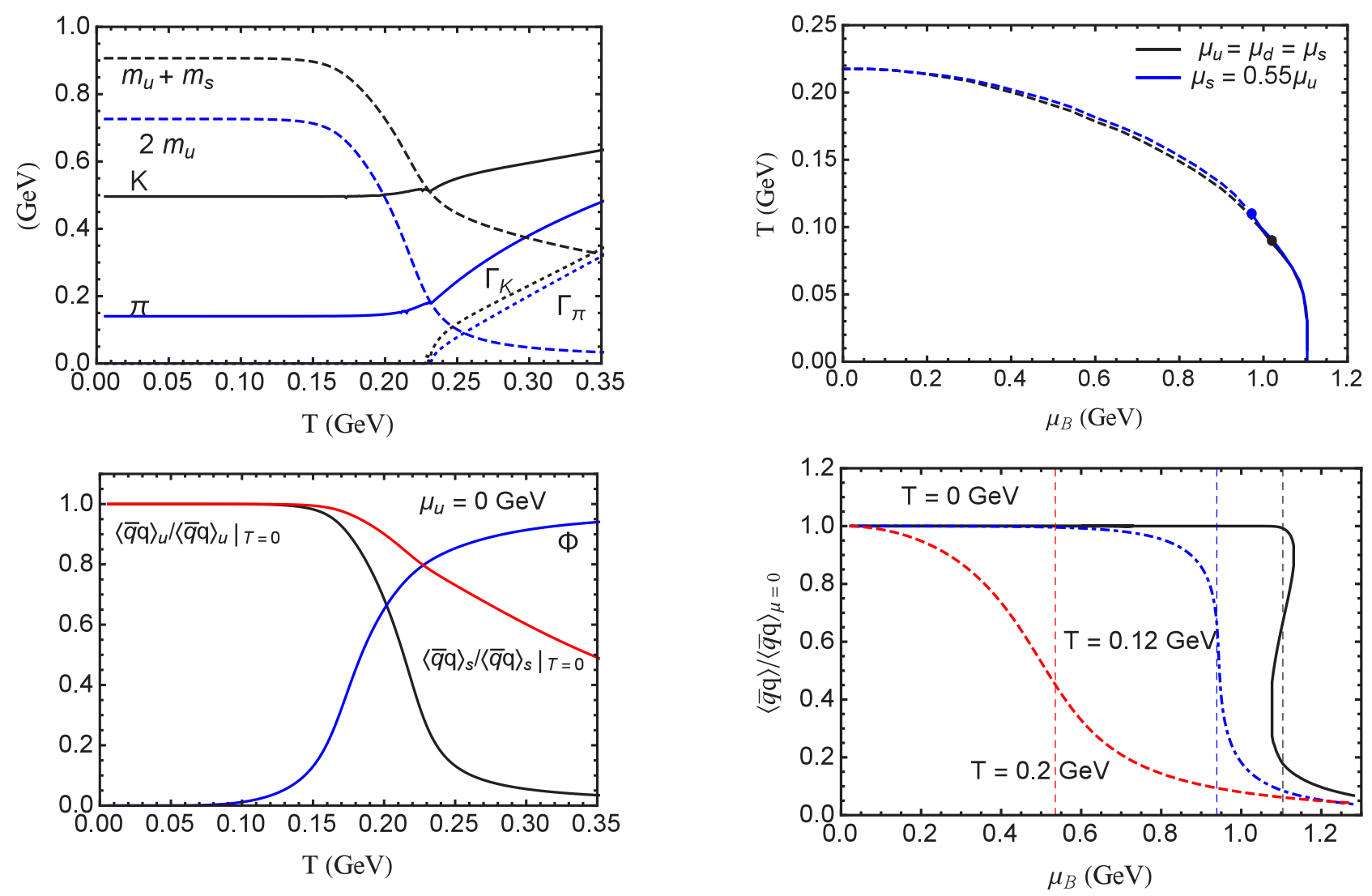

FIG. 2. (top panel) The mass spectra at $\mu_{u}=\mu_{d}=\mu_{s}=0$. The solid lines denote the meson masses: meson (black) and pion (blue), dashed lines denote $q \bar{q}$ the thresholds for the mesons, dotted lines denote the meson width (bottom panel) The quark normalized condensates of light and strange quarks and the Polyakov loop field $\Phi$.

transition is soft (crossover), the points of the crossover are defined as the local maximum of $\left.\frac{\partial\langle\bar{q} q\rangle}{\partial T}\right|_{\mu_{B}=\text { const. }}$. At low temperatures and high chemical potential, the crossover turns into the first-order transition, which can be defined as the maximum of $\left.\frac{\partial^{2} \Omega}{\partial \mu_{u}^{2}}\right|_{T=\text { const }}$ [31]. The first-order phase transition ends at the point called the critical endpoint (CEP) $\left(\mu_{B, \mathrm{CEP}}=0.99\right.$, $\left.T_{\mathrm{CEP}}=0.1\right)$ for Case I and CEP $\left(\mu_{B, \mathrm{CEP}}=0.972, T_{\mathrm{CEP}}=\right.$ 0.11 ) for Case II, the CEPs in both cases are close to each other. As can be seen, the critical temperature of the crossover transition at $\mu_{B}=0 \mathrm{GeV}$ is higher $\left(T_{c}=0.218\right)$ than predicted by lattice QCD, $T_{c}=154(9)$ [20].

In Fig. 3 (top panel), the chiral condensate as a function of the chemical potential is shown. As can be seen, at low temperature the gap equations (8) have several solutions (or a break as a function $\mu_{B}$ ) and in the system there appears the first-order transition; at high temperature (after the CEP) the quark condensate changes softly (crossover).

At nonzero chemical potential and low $T$, the splitting of mass in charged multiplets is due to excitation of the Dirac sea modified by the presence of the medium (see Fig. 4). In dense baryon matter, the concentration of light quarks is very high [32]. Therefore, the creation of an $s \bar{s}$ pair dominates because of the Pauli principle: when the Fermi energy for light quarks is higher than $s \bar{s}$ mass, the creation of the last one is energy

efficient. The increase in the $K^{+}(\bar{u} s)$ mass, with respect to these of $K^{-}(\bar{s} u)$, is justified again by the Pauli blocking for the $s$ quark (see for discussion Refs. [33-36]). Technically, to describe the mesons in dense matter, the chemical potential of quarks must be related to the Fermi momentum $\lambda_{i}, \mu_{i}=$ $\left(\lambda_{i}^{2}+m_{i}^{2}\right)^{1 / 2}$. The latter affects the limits of integration in

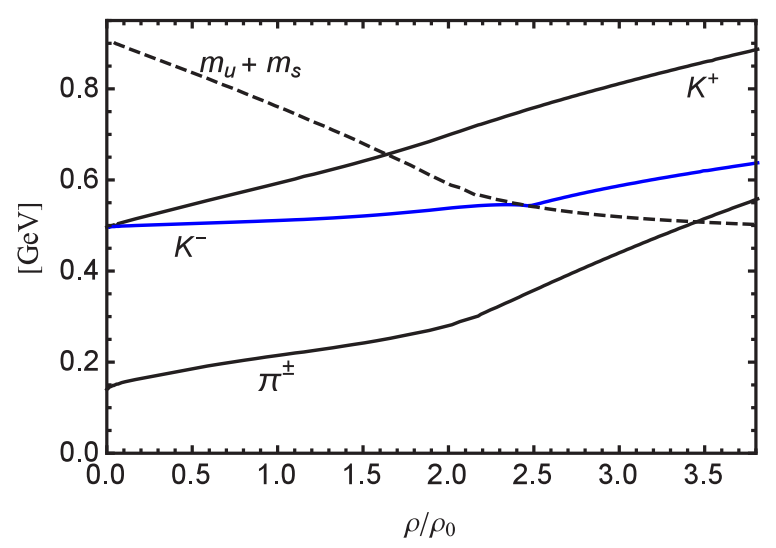

FIG. 4. The spectra of meson masses as a function of the normalized baryon density at $T=0 \mathrm{GeV}$ for Case I. 
Eqs. (16) and (17). It is clear that the pions for the chosen cases $\left(m_{u}=m_{d}\right)$ are still degenerate.

To discuss the horn problem, we have to consider the ratio of the number of kaons to the number of pions. Within the PNJL model the number densities of mesons $\left(K^{ \pm} / \pi^{ \pm}=\right.$ $\left.n_{K^{ \pm}} / n_{\pi^{ \pm}}\right)$can be calculated as

$$
\begin{aligned}
& n_{K^{ \pm}}=\int_{0}^{\infty} p^{2} d p \frac{1}{e^{\left(\sqrt{\left.p^{2}+m_{K^{ \pm}} \mp \mu_{K^{ \pm}}\right)}-1\right.}}, \\
& n_{\pi^{ \pm}}=\int_{0}^{\infty} p^{2} d p \frac{1}{e^{\left(\sqrt{p^{2}+m_{\pi^{ \pm}}} \mu_{\pi^{ \pm}}\right)}-1} .
\end{aligned}
$$

The chemical potential for pions is a phenomenological parameter and in this work it was chosen as a constant, $\mu_{\pi}=$ 0.135, following the works [37-39], but in Ref. [38] it was supposed that $\mu_{\pi}$ can depend on $T$. The chemical potential for kaons can be defined (see, for example, Refs. [39,40]) from $\mu_{q}=B_{q} \mu_{B}+S_{q} \mu_{s}+I_{q} \mu_{q}$, and in the isospin-symmetry case $\left(I_{q}=0\right)$, the result is $\mu_{K}=\mu_{u}-\mu_{s}$.

If all experimental data are taken from various experiments, it is shown in the statistical model that for each experiment the temperature and the baryon chemical potential of freeze-out [41] can be found by using the parametrization suggested by Cleymans et al. It turned out to be possible to rescale the experimental data in the variable $T / \mu_{B}$ (see Fig. 5, bottom panel), which is more suitable for theoretical calculations:

$$
\begin{aligned}
T\left(\mu_{B}\right) & =a-b \mu_{B}^{2}-c \mu_{B}^{4}, \\
\mu_{B}(\sqrt{s}) & =\frac{d}{1+e \sqrt{s}},
\end{aligned}
$$

where $a=0.166 \pm 0.002 \mathrm{GeV}, b=0.139 \pm 0.016 \mathrm{GeV}^{-1}$, $c=0.053 \pm 0.021 \mathrm{GeV}^{-3}, d=1.308 \pm 0.028 \mathrm{GeV}$, and $e=$ $0.273 \pm 0.008 \mathrm{GeV}^{-1}$.

It is evident that the experimental data at higher energy correspond to high temperature and low density or chemical potential, and the data at low energy correspond to high density or chemical potential and low temperature.

The calculated (top panel) and rescaled experimental (bottom panel) ratios $n_{K^{ \pm}} / n_{\pi^{ \pm}}$are shown in Fig. 5 (top panel) as a function of the scaling variable $T / \mu_{B}$, where the values $T$ and $\mu_{B}$ were chosen along the chiral phase-transition line, which, generally speaking, does not coincide with the freeze-out curve.

It is clearly seen from the figure that, in the region of high temperature and low density (high values of $T / \mu_{B}$ ), the $K^{+} / \pi^{+}$and $K^{-} / \pi^{-}$ratios tend to the same value. These results are in agreement with experimental results. In the PNJL model at high temperature and low density the difference between the mass of charged kaon multiplets decreases, their masses become equal to each other (kaons are degenerate at $T=0$ ), and, as can be seen, the difference in ratios can also decrease.

At low values of $T / \mu_{B}$ (high chemical potential and low temperatures) an enhancement in the $K^{+} / \pi^{+}$ratio similar to the experimental data is clearly seen. The absence of such structure in the $K^{-} / \pi^{-}$ratio can be explained by invoking the different sensibility to the medium density of the positive
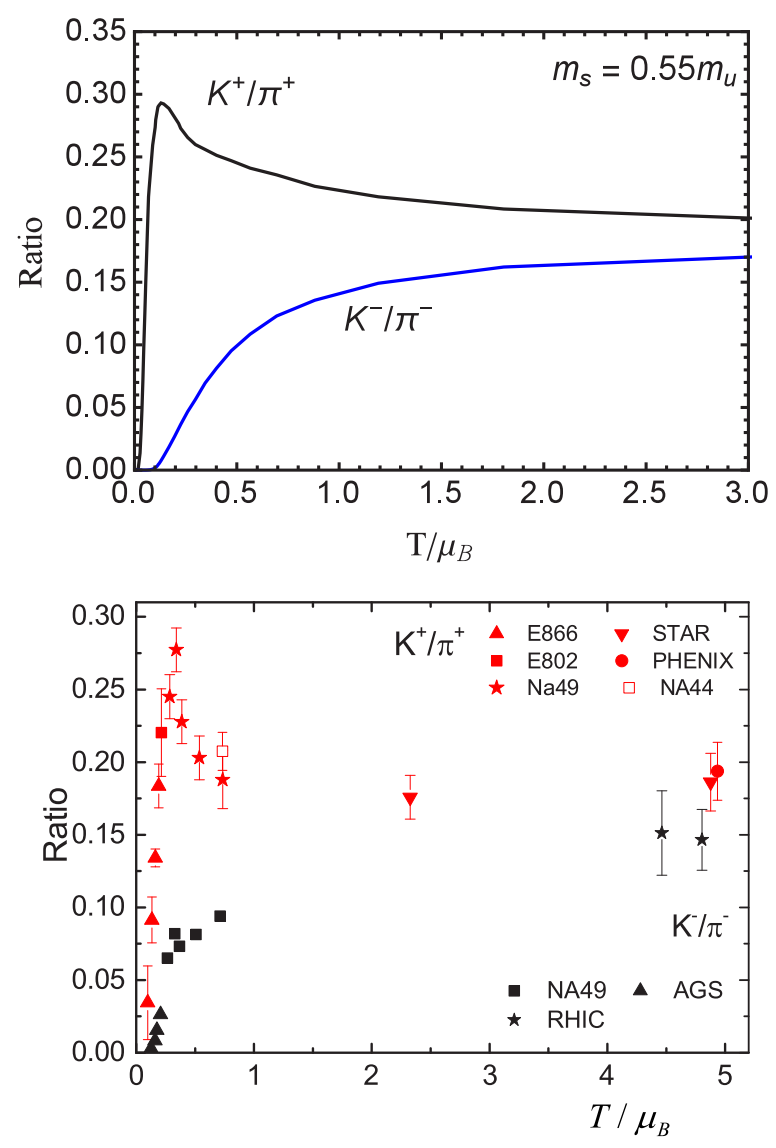

FIG. 5. (top panel) $K^{+} / \pi^{+}, K^{-} / \pi^{-}$ratio as function of $T / \mu_{B}$ for cases I and II. (bottom panel) Experimental data for $K^{+} / \pi^{+}$ratio as a function of the rescaled variable $T / \mu_{B}$.

and negative charged kaons. In time, when the relative number of baryons decreases with increasing energy, the number of negatively charged kaons will not change, opposite to the positive charged kaons, the number of which must be balanced by strange baryons and, therefore, will decrease [42].

The quark-matter properties in the frame of the PNJL model are formed by the choice of different assumptions for the environment: Case I and Case II. Generally speaking, both invented assumptions cannot reproduce the properties of the medium in a real collision of heavy ions. Nevertheless, the choice of these two cases can illustrate that the peak position is connected with the position of the critical endpoint. In Fig. 6 , it can be seen how the enhancement in the $K^{+} / \pi^{+}$ratio depends on the choice of the matter: in Case I, when $\mu_{K}=0$ (and $\mu_{S}=0$ ) there is no enhancement in the ratio. For Case II it can be seen that the value of $\mu_{s} / \mu_{u}$ affects the position and the height of the peak in the kaon to pion ratio. As can be seen in Fig. 6, the maxima are placed near the critical endpoints (vertical lines in Fig. 6). The high $K^{+} / \pi^{+}$ratio also depends on the choice of the values $T$ and $\mu_{B}$ where it is calculated. The contour lines where the $\mathrm{K}^{+} / \pi^{+}$ratio remains constant are shown in Fig. 7 on the phase-diagram plane together with the phase diagram. As can be seen, the ratio reaches the maximal value near the critical point in the region of the first-order transition and also slightly above the phase transition line. 


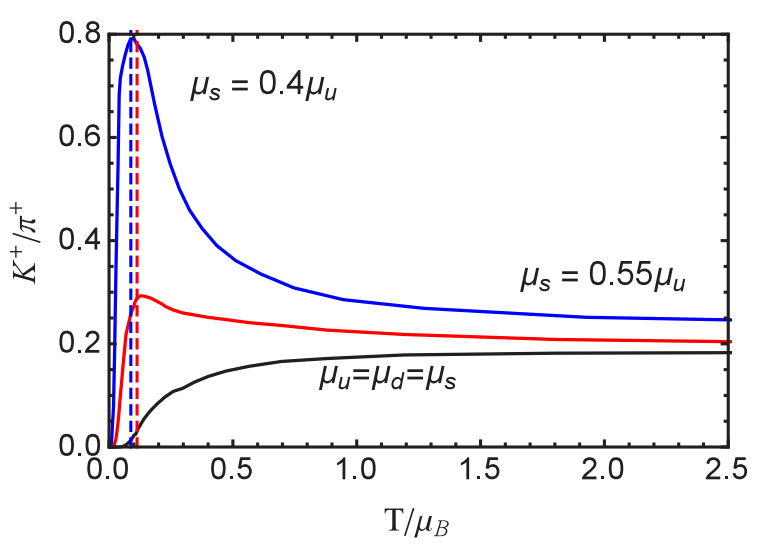

FIG. 6. The $K^{+} / \pi^{+}$ratio as a function of $T / \mu_{B}$ for the different cases I and II.

For comparison, similar lines were obtained in the statistical model [43] where the transition line is the line of freeze-out.

\section{CONCLUSION}

The strangeness enhancement in heavy-ion collisions has been suggested as the QGP signal long ago $[44,45]$. The reason is that the probability of the process $g g \rightarrow s \bar{s}$ in QGP increases due the high density of gluons and due the chiral symmetry restoration in the strange sector ( $s$ mass becomes lower). In dense matter the creation of the $s \bar{s}$ pair dominates because of the Pauli principle: a high concentration of light quarks leads to the Fermi energy for light quarks becoming higher than the $s \bar{s}$ mass and the creation of the last one is energy efficient. Therefore, if the quark-gluon plasma is created in the heavy-ion collision, the enhancement of the strangeness can be expected in comparison soon with the $p-p$ or $p$ - $n$ collision.

The dependence of the strangeness to nonstrangeness ratio was discussed in Refs. [14,43]. In the standard picture, freeze-out is interpreted as a competition between fireball expansion and the interaction of the constituents. Thus, it is

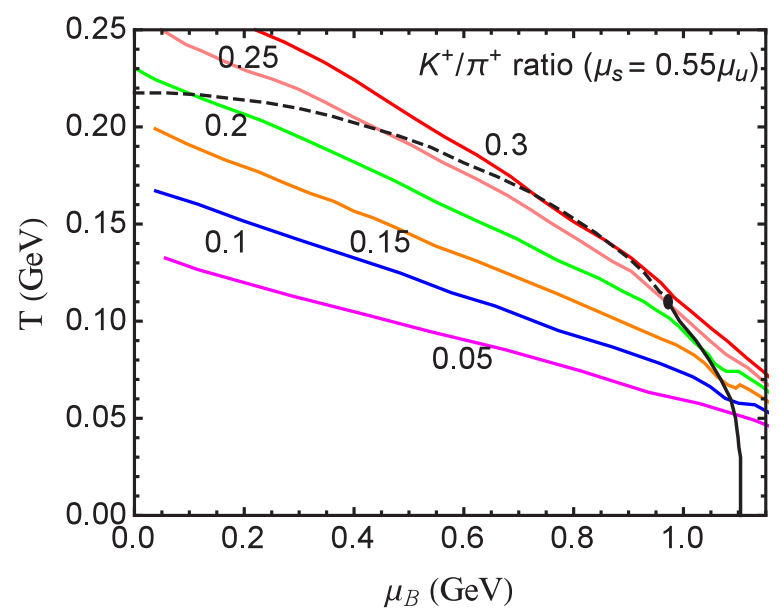

FIG. 7. The contour plot of the $K^{+} / \pi^{+}$ratio on the phase diagram (black line) plane $T-\mu_{B}$. natural to expect the system-size dependence in freeze-out conditions, since constituent interactions decrease as one goes from nucleus-nucleus $(A+A)$ to proton-nucleus $(p+A)$ and proton-proton $(p+p)$ collisions. The freeze-out hypersurface is usually extracted for three different freeze-out schemes that differ in the way strangeness is treated. The recent results [46] confirm expectations from the previous analysis of the system-size dependence in the freeze-out scheme with mean hadron yields: while heavy-ion collisions are dominated by constituent interactions, smaller collision systems like proton + nucleus and proton + proton collisions with lesser constituent interaction prefer a unified freeze-out scheme with varying degrees of strangeness equilibration.

The main idea of this work was to show that the cause of the appearance of the horn at energies $\sqrt{s_{N N}}=8-10 \mathrm{GeV}$ may be a qualitative change in the state of the environment where kaons and pions are created. In the work [14] the quick increase in the $K^{+} / \pi^{+}$ratio and its decrease with further increasing energy was interpreted as a sequence of the chiralsymmetry restoration effect and the deconfinement effect. In the PNJL model the picture is the following (Fig. 3): when $T$ and $\mu_{B}$ are chosen on the phase diagram line, the system is in the phase-transition region and the chiral condensate is still not destroyed. The main difference between the choice of $T$ and $\mu_{B}$ along the line is whether we are in the crossover region or in the first-order transition region (vertical lines in Fig. 3, bottom panel). In the region of the first-order transition (low temperatures) the value of $\Phi \rightarrow 0$ and the matter is confined. In the region of the crossover deconfinement transition takes place.

In the PNJL model, the masses of positive and negative mesons are split at high densities. This splitting can explain the difference in the behavior of the $K / \pi$ ratios for different charge signs in the high-density region and the fact that they tend to the same value at high temperatures and low densities, where kaons become degenerate. It was shown that, in Case I (when the chemical potential of the strange quark coincides with the chemical potential of the light quark and there is no strange chemical potential in the system), the enhancement in the $K^{+} / \pi^{+}$ratio is absent. This can be a signal that the peak is sensitive to the properties of the matter. Therefore, in future work, one can check the presence or absence of such a peak in different media (a medium with an equal baryon density, a medium with beta equilibrium, strange matter, or a medium with an equal number of protons, neutrons, hyperons, etc.). The second interesting result was that the region of maximum values in the ratio is localized near the critical endpoint. This hypothesis can also be verified by including a vector interaction in the PNJL model, which can allow one to move the critical point up to its removing from the phase diagram.

\section{ACKNOWLEDGMENTS}

We are thankful to A. Khvorostukhin and E. Kolomeitsev for useful advice. We also thank W. Cassing and D. Blaschke for discussions. The work of A.F. was supported by the Russian Science Foundation under Grant No. 17-12-01427. 
[1] S. V. Afanasiev et al. (NA49 Collabration), Phys. Rev. C 66, 054902 (2002).

[2] STAR BES White paper, SN0598.

[3] C. Alt et al. (NA49 Collaboration), Phys. Rev. C 77, 024903 (2008).

[4] J. L. Klay et al. (E895 Collaboration), Phys. Rev. C 68, 054905 (2003)

[5] B. I. Abelev et al. (STAR Collaboration), Phys. Rev. C 81, 024911 (2010); M. M. Aggarwal et al., ibid. 83, 024901 (2011).

[6] C. Adler et al. (STAR Collaboration), Phys. Lett. B 595, 143 (2004).

[7] M. Kliemant, B. Lungwitz, and M. Gaździcki, Phys. Rev. C 69, 044903 (2004).

[8] W. Ehehalt and W. Cassing, Nucl. Phys. A 602, 449 (1996).

[9] W. Cassing and E. L. Bratkovskaya, Phys. Rep. 308, 65 (1999).

[10] H. Petersen, M. Bleicher, S. A. Bass, and H. Stöcker, arXiv:0805.0567.

[11] J. Cleymans, E. Suhoen, and G. M. Weber, Z. Phys. C: Part. Fields 53, 485 (1992).

[12] H. Sorge, Phys. Rev. C 52, 3291 (1995).

[13] M. Gazdzicki and M. I. Gorenstein, Acta Phys. Pol. B 30, 2705 (1999); 42, 307 (2011).

[14] A. Palmese, W. Cassing, E. Seifert, T. Steinert, P. Moreau, and E. L. Bratkovskaya, Phys. Rev. C 94, 044912 (2016).

[15] T. D. Cohen, R. J. Furnstahl, and D. K. Griegel, Phys. Rev. C 45, 1881 (1992).

[16] A. Andronic, P. Braun-Munzinger, and J. Stachel, Phys. Lett. B 673, 142 (2009).

[17] K. Fukushima, Phys. Rev. D 77, 114028 (2008).

[18] P. Costa, M. C. Ruivo, C. A. de Sousa, H. Hansen, and W. M. Alberico, Phys. Rev. D 79, 116003 (2009).

[19] E. Blanquier, J. Phys. G 38, 105003 (2011).

[20] A. Bazavov et al. (HotQCD Collaboration), Phys. Rev. D 85, 054503 (2012).

[21] A. V. Friesen, Y. L. Kalinovsky, and V. D. Toneev, Int. J. Mod. Phys. A 27, 1250013 (2012).

[22] A. V. Friesen, Y. L. Kalinovsky, and V. D. Toneev, Int. J. Mod. Phys. A 30, 1550089 (2015).

[23] R. D. Pisarski, Phys. Rev. D 62, 111501(R) (2000).
[24] C. Ratti, M. A. Thaler, and W. Weise, Phys. Rev. D 73, 014019 (2006).

[25] A. M. Polyakov, Phys. Lett. B 72, 477 (1978).

[26] G. Boyd, J. Engels, F. Karsch, E. Laermann, C. Legeland, M. Lütgemeier, and B. Petersson, Nucl. Phys. B 469, 419 (1996).

[27] S. Rössner, C. Ratti, and W. Weise, Phys. Rev. D 75, 034007 (2007).

[28] S. P. Klevansky, Rev. Mod. Phys. 64, 649 (1992).

[29] P. Rehberg, S. P. Klevansky, and J. Hüfner, Phys. Rev. C 53, 410 (1996).

[30] D. Blaschke, A. Dubinin, A. Radzhabov, and A. Wergieluk, Phys. Rev. D 96, 094008 (2017).

[31] T. Kunihiro, Phys. Lett. B 219, 363 (1989).

[32] J. Stachel and G. R. Young, Annu. Rev. Nucl. Part. Sci. 42, 537 (1992).

[33] M. Lutz, A. Steiner, and W. Weise, Nucl. Phys. A 574, 755 (1994).

[34] M. C. Ruivo and C. A. de Sousa, Phys. Lett. B 385, 39 (1996).

[35] P. Costa, M. C. Ruivo, and Y. L. Kalinovsky, Phys. Lett. B 560, 171 (2003).

[36] P. Costa, M. C. Ruivo, C. A. de Sousa, and Y. L. Kalinovsky, Phys. Rev. C 70, 025204 (2004).

[37] M. Kataja and P. V. Ruuskanen, Phys. Lett. B 243, 181 (1990).

[38] V. Begun, W. Florkowski, and M. Rybczynski, Phys. Rev. C 90, 014906 (2014).

[39] M. Naskret, D. Blaschke, and A. Dubinin, Phys. Part. Nucl. 46, 789 (2015).

[40] A. Lavagno and D. Pigato, EPJ Web Conf. 37, 09022 (2012).

[41] J. Cleymans, H. Oeschler, K. Redlich, and S. Wheaton, Phys. Rev. C 73, 034905 (2006).

[42] J. Cleymans, H. Oeschler, K. Redlich, and S. Wheaton, J. Phys.: Conf. Ser. 50, 406 (2006).

[43] H. Oeschler, J. Cleymans, K. Redlich, and S. Wheaton, PoS CPOD2009, 032 (2009).

[44] J. Rafelski and B. Muller, Phys. Rev. Lett. 48, 1066 (1982).

[45] P. Koch, B. Muller, and J. Rafelski, Phys. Rep. 142, 167 (1986).

[46] A. K. Dash, R. Singh, S. Chatterjee, C. Jena, and B. Mohanty, Phys. Rev. C 98, 064902 (2018). 